\title{
Derivation and comparison of model equations for interfacial capillary-gravity waves in deep water
}

\author{
Henrik Kalisch \\ Department of Mathematics, University of Bergen, Norway
}

Available online 28 November 2006

\begin{abstract}
A matched asymptotic expansion is used to give a formal derivation of a number of systems of model equations for the evolution of interfacial waves subject to capillarity. For one of these systems, approximate solitary waves are found numerically, and the solutions are compared to the Benjamin equation which arises in the special case of one-way propagation.

(C) 2006 IMACS. Published by Elsevier B.V. All rights reserved.
\end{abstract}

Keywords: Capillary-gravity waves; Internal waves; Solitary waves; Long-wave approximation; Benjamin equation

\section{Introduction}

The object of this note is the systematic derivation of a number of model equations which are of use in the description of the evolution of long-crested internal waves in two-fluid systems. In particular, it is our purpose to give a derivation of the so-called Benjamin equation

$$
u_{t}+u_{x}+u u_{x}-\mathcal{H} u_{x x}-\tau u_{x x x}=0
$$

which appears as a one-dimensional model for interfacial waves if one of the layers is thin, the other is deep, and the interface is subject to capillarity. In this equation, $\mathcal{H}$ denotes the Hilbert transform, and $\tau$ is a positive parameter measuring the strength of interfacial tension as explained by Benjamin in Ref. [5]. Interest in this equation stems from wave tank experiments as reported by Koop and Butler [18] and others. The regime of applicability of this equation is a somewhat narrow range, which fact is explained in more detail by Albert et al. in Ref. [3]. The equation was also derived by Tuck and Wiryanto [27] in the case when $\tau<0$ represents higher order gravity effects. In this situation, the equation has been termed the composite long-wave equation. One crucial assumption in the Benjamin equation is that the principal direction of propagation of the waves is to the right. Here, we take a somewhat broader view and allow waves to travel in both directions. The resulting equations are systems of two coupled evolution equations, similar to Boussinesq-type systems describing waves at the fluid surface.

In the case when capillarity can be neglected, similar model equations have been obtained by Choi and Camassa [8,9], and also by Craig et al. [12,13], Matsuno [21] and Ostrovsky and Grue [23]. There have also been studies of interfacial capillary-gravity waves, mostly in the context of steady waves. For example, the papers by Christodoulides and Dias [10], Dias and Iooss [14], Laget and Dias [19], and Sha and Vanden-Broeck [26] contain studies leaning on numerical approximation of the Euler equations and bifurcation analysis.

E-mail address: henrik.kalisch@mi.uib.no. 


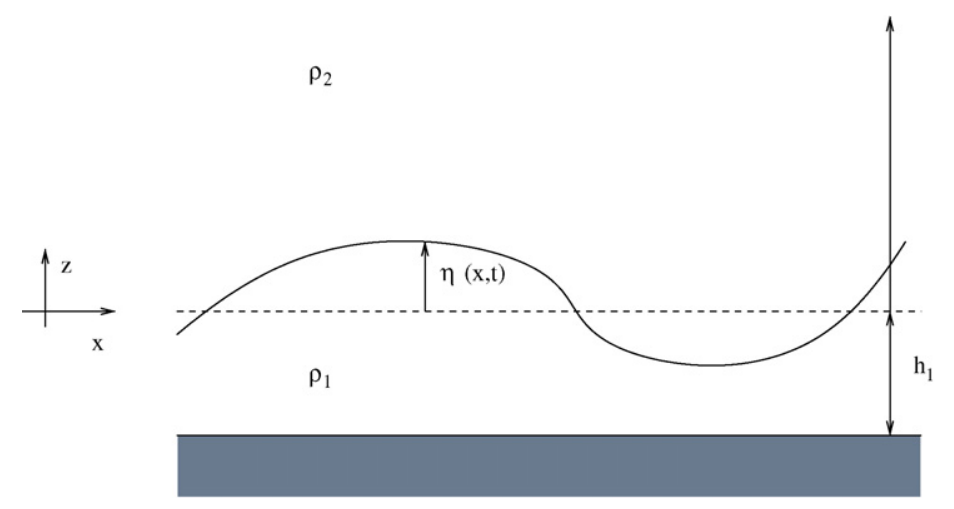

Fig. 1. A two-fluid system.

The problem under study here is the description of the evolution of a two-layer system of fluids divided by a sharp interface. This problem exhibits the well known Kelvin-Helmholtz instability for shear flow. According to the work of Ebin [15], this instability manifests itself in the fact that the initial-value problem for the full Euler equations with a sharp interface is ill-posed in the Hadamard sense. Since many of the above mentioned model equations are also unstable, or even ill-posed, capillarity can be seen as a way to stabilize the equations. It should be pointed out that the present analysis does not include proofs of convergence of solutions of the aforementioned systems of equations to solutions of the full Euler equations. Such proofs have been provided in some cases for model equations for surface waves by Craig [11], Schneider and Wayne [24,25], and Bona et al. [7]. However, for interfacial waves, such an analysis seems out of reach at the present time.

The remainder of the introduction is devoted to the description of the basic equations governing the fluid motion and the discussion of the linear dispersion relation in the situation at hand. In Section 2, the derivation of the evolutions equations is given, and in Section 3 a number of other equations including one-way models such as (1.1) are derived. Finally, in Section 4, we present a numerical study of one of the systems appearing in Section 2, and compare it to the Benjamin Eq. (1.1).

The geometry of the system under study has been sketched in Fig. 1. The fluid in the lower layer has density $\rho_{1}$, while the one in the upper layer has density $\rho_{2}$, where it is assumed that $\rho_{2}<\rho_{1}$. At rest, the depth of the lower layer is $h_{1}$, measured from the flat bottom located at $z=-h_{1}$. The upper layer is infinitely deep, and the interface between the fluids is subject to interfacial tension proportional to its curvature with a proportionality constant $\tau$. When viscosity is neglected, the basic continuum model is given by a pair of Euler equations coupled through boundary conditions at the common free interface. Irrationality is also assumed, so that the Euler equations can be written in terms of velocity potentials $\psi$ in the upper layer and $\phi$ in the lower layer. Moreover, it is assumed that the interface between the two layers can be described by a function $\eta(x, t)$, measuring the deflection of the interface from its rest position. The equations take the form

$$
\begin{array}{ll}
\triangle \psi=0, & \text { in } \eta<z<\infty, \\
\psi_{z}=0, & \text { if } z \rightarrow \infty \\
\triangle \phi=0, & \text { in }-h_{1}<z<\eta, \\
\phi_{z}=0, & \text { at } z=-h_{1} .
\end{array}
$$

At the interface, the following boundary conditions are in force:

$$
\begin{aligned}
& \eta_{t}+\psi_{x} \eta_{x}=\psi_{z}, \quad \text { at } z=\eta . \\
& \eta_{t}+\phi_{x} \eta_{x}=\phi_{z}, \quad \text { at } z=\eta . \\
& p_{1}-p_{2}=-\tau \eta_{x x}, \quad \text { at } z=\eta .
\end{aligned}
$$

The assumption of infinite depth is useful in situations where the depth is much larger than the wavelength of a typical wave. Note that it is assumed that there is no significant variation in the direction transverse to the propagation of the waves, so that $y$-derivatives may be safely ignored. In particular, the Laplacian operator in Eqs. (1.2) and (1.3) is two-dimensional. 
The dynamic boundary condition (1.6) involving the pressures $p_{2}$ and $p_{1}$ in the upper and lower layers, respectively, is posed under the assumption that the slope of the free interface varies slowly, as the second derivative is then a good approximation to the curvature of the interface. As the focus in this work is on long waves, this is certainly a reasonable assumption. Using Bernoulli's law

$$
\frac{p}{\rho}=-\phi_{t}-\frac{1}{2}\left(\phi_{x}^{2}+\phi_{z}^{2}\right)-g z
$$

in each layer, the third condition may be rewritten as

$$
\rho_{1}\left(g \eta+\phi_{t}+\frac{1}{2} \phi_{x}^{2}+\frac{1}{2} \phi_{z}^{2}\right)-\rho_{2}\left(g \eta+\psi_{t}+\frac{1}{2} \psi_{x}^{2}+\frac{1}{2} \psi_{z}^{2}\right)=\tau \eta_{x x} .
$$

If Eqs. (1.2)-(1.6) are linearized, and oscillatory solutions of the form $\mathrm{e}^{\mathrm{i} k x-\mathrm{i} \omega t}$ are sought, then the dispersion relation

$$
\omega^{2}(k)=k \frac{\left(\rho_{1}-\rho_{2}\right) g+\tau k^{2}}{\rho_{1} \operatorname{coth}\left(k h_{1}\right)+\rho_{2} \operatorname{sgn}(k)}
$$

with phase speed

$$
c^{2}(k)=\frac{\left(\rho_{1}-\rho_{2}\right) g+\tau k^{2}}{\rho_{1} k \operatorname{coth}\left(k h_{1}\right)+\rho_{2}|k|}
$$

may be recovered as shown in Ref. [20]. An asymptotic expansion in $|k|$ gives

$$
c^{2}(k)=g h_{1}\left(1-\frac{\rho_{2}}{\rho_{1}}\right)\left[1-\frac{\rho_{2}}{\rho_{1}} h_{1}|k|+\left\{\frac{\tau}{g\left(\rho_{1}-\rho_{2}\right)}+\left(\frac{\rho_{2}^{2}}{\rho_{1}^{2}}-\frac{1}{3}\right) h_{1}^{2}\right\} k^{2}\right]+O\left(|k|^{3}\right) .
$$

For long waves, $|k|^{2}$ is sufficiently smaller than $|k|$ so that the term in brackets can be neglected. However, if it is assumed that $\rho_{1}$ and $\rho_{2}$ are close and $\tau k^{2} / g\left(\rho_{2}-\rho_{1}\right) \sim h_{1}|k|$, then capillarity should be included, and the corresponding linear equation is

$$
\eta_{t t}-g h_{1}\left(1-\frac{\rho_{2}}{\rho_{1}}\right)\left[\eta_{x x}-h_{1} \frac{\rho_{2}}{\rho_{1}} H \eta_{x x x}-\frac{\tau}{g\left(\rho_{1}-\rho_{2}\right)} \eta_{x x x x}\right]=0 .
$$

Before we carry on, it should be mentioned that the geometry of the fluid system under study can be changed by a simple change of variables. In particular, the change of variables

$$
g \rightarrow-g \quad \eta \rightarrow-\eta \quad \rho_{1} \leftrightarrow \rho_{2}
$$

will yield a system with a thin upper layer and a deep lower layer. In this case, the Neumann boundary condition at the bottom becomes the rigid-lid assumption at the surface. The situation of a coupled surface-interface model is not treated in the present article. The reader may consult [13] for comments on this case.

\section{Derivation of the evolution equations}

Eq. (1.9) is valid only for waves with infinitesimally small amplitude. To allow for the description of a wider range of waves, finite-amplitude effects must be considered. The goal is to obtain an equation which provides a good model for waves in a regime where nonlinear and dispersive effects are balanced. The usual point of view (cf. [6,28]) is to consider dispersive and nonlinear effects as small corrections to the basic linear hyperbolic system

$$
\begin{aligned}
& w_{t}+C_{0}^{2} \eta_{x}=0, \\
& \eta_{t}+w_{x}=0,
\end{aligned}
$$

with wavespeed $C_{0}$. Here $C_{0}=c_{0} v_{0}$, where $c_{0}=\sqrt{g h_{1}}$ is the usual limiting long-wave speed for surface waves on homogeneous-layer flows, and $v_{0}=\sqrt{1-\left(\rho_{2} / \rho_{1}\right)}$ is a dimensionless quantity. Such a simplified model will be valid only for small, long waves, and over a limited time scale.

As mentioned in the introduction, the significant quantities are the amplitude $a$ and the wavelength $\lambda$ of a typical wave. To quantify the meaning of 'small' and 'long', the depth $h_{1}$ of the lower layer is selected as a natural spatial 
scale. We then introduce the scaling factors $\sigma=a / h_{1}$ and $\epsilon=h_{1} / \lambda$ which are assumed to be small and of the same order. To quantify the interfacial tension, the parameter $\mu=\tau / g\left(\rho_{1}-\rho_{2}\right) \lambda^{2}$ is introduced. In order to include capillary effects in the description, it will also be assumed that $\mu$ is of the same order as $\sigma$ and $\epsilon$. Note that the usual definition of the Bond number does not apply in this context, because the total depth of the fluid is infinite. Instead, one may define a quantity by comparing $\mu$ to $\epsilon$, thus effectively measuring the influence of capillarity versus gravity. Our set of assumptions include the supposition that the effects of gravity and capillarity are balanced.

The derivation of the model equations is based upon the observation that to first order in $\epsilon, \sigma$ and $\mu$ the problem is linear in the upper layer. We thus have to solve only a linear elliptic problem in the upper layer, with boundary conditions subject to matching the lower layer. To make the difference in the $z$-scales in the two layers explicit, two different normalizations for the $z$-variable are used. For the sake of notational convenience, the original variables appear henceforth with a prime.

$$
\begin{aligned}
& z^{\prime}=\lambda Z, \quad \text { in } \eta^{\prime}<z^{\prime}<\infty, \\
& z^{\prime}=h_{1} z, \quad \text { in }-h_{1}<z^{\prime}<\eta^{\prime} .
\end{aligned}
$$

The other variables are nondimensionalized as follows:

$$
x^{\prime}=\lambda x, \quad t^{\prime}=\frac{\lambda t}{c_{0} v_{0}}, \quad \eta^{\prime}=\frac{a}{v_{0}} \eta, \quad \phi^{\prime}=\frac{g \lambda a}{c_{0}} v_{0} \phi, \quad \psi^{\prime}=\frac{g \lambda a}{c_{0}} v_{0} \psi,
$$

where $c_{0}=\sqrt{g h_{1}}$ and $v_{0}=\sqrt{1-\left(\rho_{2} / \rho_{1}\right)}$ as before. The full equations in the new variables are

$$
\begin{array}{ll}
\psi_{x x}+\psi_{Z Z}=0, & \text { in } \epsilon \sigma \eta<Z, \\
\psi_{Z}=0, & \text { if } Z \rightarrow \infty, \\
\epsilon^{2} \phi_{x x}+\phi_{z z}=0, & \text { in }-1<z<\sigma \eta, \\
\phi_{z}=0, & \text { at } z=-1 .
\end{array}
$$

Subtracting the kinematic boundary conditions (1.4), (1.5) at the interface, and using the original primed variables yields

$$
\phi_{z^{\prime}}^{\prime}=\psi_{z^{\prime}}^{\prime}+\eta_{x^{\prime}}^{\prime}\left(\phi_{x^{\prime}}^{\prime}-\psi_{x^{\prime}}^{\prime}\right)
$$

In the new variables, this becomes

$$
\phi_{z}=\epsilon \psi_{Z}+\epsilon^{2} \sigma \eta_{x}\left(\phi_{x}-\psi_{x}\right) .
$$

Thus, the matching condition at the interface is

$$
\psi_{Z}=\frac{1}{\epsilon} \phi_{z}+O(\epsilon \sigma), \quad \text { at } Z=\epsilon \sigma \eta .
$$

The strategy for deriving approximate equations is to find expansions for $\psi$ and $\phi$ subject to (2.4), and to substitute the expansions into the boundary conditions (1.5) and (1.7). Disregarding terms of higher order in $\epsilon, \sigma$, and $\mu$ will then give a pair of equations approximating the motion of the interface. First, we focus on the velocity potential $\phi$ in the lower layer. Since the lower layer is relatively thin, it is assumed that $\phi$ can be written as an expansion in the small parameter $\epsilon$, namely

$$
\phi=\sum_{k=0}^{\infty} \epsilon^{k}(z+1)^{k} f_{k}(x, t) .
$$

Laplace's equation and the boundary condition at the bottom of the lower layer give

$$
\phi=\sum_{k=0}^{\infty}(-1)^{k} \epsilon^{2 k} \frac{(z+1)^{2 k}}{(2 k) !} \frac{\partial^{k} f_{0}}{\partial x^{2}}(x, t)=f-\epsilon^{2} \frac{(z+1)^{2}}{2} f_{x x}+O\left(\epsilon^{4}\right),
$$

where $f_{0}$ is now called $f$. This expression for $\phi$ gives

$$
\phi_{z}=-\epsilon^{2}(z+1) f_{x x}+O\left(\epsilon^{4}\right) .
$$


Next, a representation for $\psi$ in terms of $\phi$ will be sought. The potential $\psi$ satisfies Laplace's equation in the upper layer with a boundary condition given by matching with the lower layer at the interface. Combining (2.4) and (2.5) yields the problem

$$
\begin{array}{ll}
\Delta \psi=0, & \text { in } Z>\epsilon \sigma \eta, \\
\psi_{Z}=-\epsilon(1+\sigma \eta) f_{x x}+O\left(\epsilon^{2}, \epsilon \sigma\right), & \text { at } Z=\epsilon \sigma \eta .
\end{array}
$$

The boundary condition shows that $\psi$ is of order $\epsilon$. Moreover, $\psi_{t}$ also satisfies Laplace's equation, so that when terms of $O\left(\epsilon^{2}\right)$ and $O(\epsilon \sigma)$ are neglected, the following elliptic problem appears:

$$
\begin{array}{ll}
\Delta \psi_{t}=0, & \text { in } Z>0, \\
\psi_{t Z}=-\epsilon f_{x x t}, & \text { at } Z=0 .
\end{array}
$$

The solution of this latter boundary-value problem is given in terms of the Poisson kernel $P$ and the Hilbert transform $\mathcal{H}$ as

$$
\psi_{t}=-\epsilon \mathcal{H}\left(\partial_{x}^{-1} P(z) f_{x x t}\right) .
$$

Consequently, it is immediate that

$$
\psi_{t}(Z=\epsilon \sigma \eta)=-\epsilon \mathcal{H} f_{x t}+O\left(\epsilon^{2}, \epsilon \sigma\right) .
$$

Note that the Hilbert transform is defined be the following singular integral:

$$
\mathcal{H} f(x)=\operatorname{p.v} \cdot \frac{1}{\pi} \int_{-\infty}^{\infty} \frac{f(x-y)}{y} \mathrm{~d} y .
$$

We are now in a position to derive a set of evolution equations by inserting the expressions for $\phi$ and $\psi$ into the boundary conditions (1.7) and (1.5). In normalized variables the boundary condition (1.7) is written as

$$
\rho_{1}\left(\eta+v_{0}^{2} \phi_{t}+\frac{1}{2} \sigma v_{0}^{2} \phi_{x}^{2}+\frac{1}{2} \frac{\sigma}{\epsilon^{2}} v_{0}^{2} \phi_{z}^{2}\right)=\rho_{2}\left(\eta+v_{0}^{2} \psi_{t}+\frac{1}{2} \sigma v_{0}^{2} \psi_{x}^{2}+\frac{1}{2} \sigma v_{0}^{2} \psi_{Z}^{2}\right)+\frac{\tau}{g \lambda^{2}} \frac{1}{C_{0}^{2}} \eta_{x x} .
$$

Substituting the expansion for $\phi$ and the expression for $\psi_{t}$ and keeping terms up to $O(\epsilon, \sigma)$ gives

$$
\eta+f_{t}+\frac{1}{2} \sigma f_{x}^{2}=-\epsilon \frac{\rho_{2}}{\rho_{1}} \mathcal{H} f_{t x}+\frac{\tau}{g \rho_{1} \lambda^{2}} \frac{1}{v_{0}^{2}} \eta_{x x}+O\left(\epsilon^{2}, \epsilon \sigma\right) .
$$

Differentiating with respect to $x$, setting $w=f_{x}$ and using $\mu=\tau / g\left(\rho_{1}-\rho_{2}\right) \lambda^{2}$, there obtains

$$
\eta_{x}+w_{t}+\sigma w w_{x}+\epsilon \frac{\rho_{2}}{\rho_{1}} \mathcal{H} w_{t x}-\mu \eta_{x x x}=O\left(\epsilon^{2}, \epsilon \sigma\right) .
$$

In particular, it follows from (2.6) that $\eta_{x}+w_{t}=O(\epsilon, \mu, \sigma)$. If it is assumed that differentiation and application of the Hilbert transform do not alter the order of this relation (cf. [6]), it may be used to find

$$
w_{t}+\eta_{x}+\sigma w w_{x}-\epsilon \frac{\rho_{2}}{\rho_{1}} \mathcal{H} \eta_{x x}-\mu \eta_{x x x}=O\left(\epsilon^{2}, \epsilon \sigma, \epsilon \mu\right) .
$$

This is the first equation of the system. The second equation is obtained in the usual way (cf. [28]) from the kinematic boundary condition. In the new variables, (1.5) becomes

$$
\eta_{t}+\sigma \phi_{x} \eta_{x}-\frac{1}{\epsilon^{2}} \phi_{z}=0, \quad \text { at } y=\sigma \eta
$$

Inserting the expansion for $\phi$, there appears

$$
\eta_{t}+\sigma\left(f_{x}+O\left(\epsilon^{2}\right)\right) \eta_{x}+(1+\sigma \eta) f_{x x}=O\left(\epsilon^{2}, \epsilon \sigma\right) .
$$

Finally, substituting $w=f_{x}$ gives

$$
\eta_{t}+w_{x}+\sigma(w \eta)_{x}=O\left(\epsilon^{2}, \epsilon \sigma\right) .
$$


Disregarding terms of $O\left(\epsilon^{2}\right), O(\epsilon \sigma)$ and $O(\epsilon \mu)$, the system

$$
\begin{aligned}
& w_{t}+\eta_{x}+\sigma w w_{x}-\epsilon \frac{\rho_{2}}{\rho_{1}} \mathcal{H} \eta_{x x}-\mu \eta_{x x x}=0, \\
& \eta_{t}+w_{x}+\sigma(w \eta)_{x}=0
\end{aligned}
$$

appears as an approximate equation for waves which are governed by (1.2)-(1.6) in the case when the amplitude $a$ and the wavelength $\lambda$ satisfy the conditions set forth in the beginning of this section. The linearized equation corresponding to $(2.7)$ is

$$
\eta_{t t}-\eta_{x x}+\epsilon \frac{\rho_{2}}{\rho_{1}} \mathcal{H} \eta_{x x x}+\mu \eta_{x x x x}=0 .
$$

After rescaling to the original variables and remembering that $\epsilon=h_{1} / \lambda$ and $\mu=\tau / g\left(\rho_{1}-\rho_{2}\right) \lambda^{2}$, this equation becomes precisely the same as (1.9), which was derived using different methods. The fact that they agree lends additional credibility to the foregoing development.

\section{Other model equations}

In the previous section, it was shown how to derive from the Euler equations a simple system of equations governing the wave motion at the interface between two fluids when the waves that are to be described have a typical amplitude $a$ and a typical wavelength $\lambda$, and when the quantities $\sigma=a / h_{1}, \epsilon=h_{1} / \lambda$ and $\mu=\tau / g\left(\rho_{1}-\rho_{2}\right) \lambda^{2}$ are small and of the same order. It was also seen that the functions $\eta$ and $w$ appearing as the dependent variables in the system derived in the previous section satisfy the relation $\eta_{x}+w_{t}=O(\epsilon, \mu, \sigma)$, which was used in the derivation of (2.7). However, for some purposes it may be more convenient to keep the first equation in its original form. The resulting system

$$
\begin{aligned}
& w_{t}+\eta_{x}+\sigma w w_{x}+\epsilon \frac{\rho_{2}}{\rho_{1}} \mathcal{H} w_{t x}-\mu \eta_{x x x}=0, \\
& \eta_{t}+w_{x}+\sigma(w \eta)_{x}=0
\end{aligned}
$$

is formally equivalent to (2.7). In case interfacial tension can be neglected, the third-order derivative drops out of the equation. The system then takes the form

$$
\begin{aligned}
& w_{t}+\eta_{x}+\sigma w w_{x}+\epsilon \frac{\rho_{2}}{\rho_{1}} \mathcal{H} w_{t x}=0, \\
& \eta_{t}+w_{x}+\sigma(w \eta)_{x}=0 .
\end{aligned}
$$

Sometimes it is possible to restrict the model to waves propagating in one direction only. In this situation, a single equation suffices to describe the wavemotion. Such an equation can be extracted from the system (2.7). For the sake of notational convenience, it is assumed that $\epsilon=\sigma=\mu$. We are interested in solutions to (2.7) which propagate in the direction of increasing values of $x$. Solutions to the linear hyperbolic system (2.1) with $C_{0}=1$ propagate to the right, i.e. in the direction of increasing values of $x$, if and only if $w=\eta$. Therefore, to find solutions of (2.7) which move to the right to first order in $\epsilon$, we propose solutions of the form $w=\eta+\epsilon A$, where $A$ is a function of $\eta$ and its spatial derivatives. Substituting into (2.7), the system

$$
\begin{aligned}
& \eta_{t}+\eta_{x}+\epsilon\left(\eta \eta_{x}+A_{t}-\frac{\rho_{2}}{\rho_{1}} \mathcal{H} \eta_{x x}-\eta_{x x x}\right)=O\left(\epsilon^{2}\right), \\
& \eta_{t}+\eta_{x}+\epsilon\left(2 \eta \eta_{x}+A_{x}\right)=O\left(\epsilon^{2}\right),
\end{aligned}
$$

appears. Since $A$ is a function of $\eta$ and its derivatives, we may replace $A_{t}$ by $-A_{x}$ to order $\epsilon^{2}$. Then, for the two equations to be consistent, we need

$$
A=-\frac{1}{4} \eta^{2}-\frac{1}{2} \frac{\rho_{2}}{\rho_{1}} \mathcal{H} \eta_{x}-\frac{1}{2} \eta_{x x}
$$

Hence

$$
w=\eta-\epsilon \frac{1}{4} \eta^{2}-\epsilon \frac{1}{2} \frac{\rho_{2}}{\rho_{1}} \mathcal{H} \eta_{x}-\epsilon \frac{1}{2} \eta_{x x} .
$$


Substituting this expression into the second equation, and disregarding terms of higher order yields

$$
\eta_{t}+\eta_{x}+\epsilon \frac{3}{2} \eta \eta_{x}-\epsilon \frac{1}{2} \frac{\rho_{2}}{\rho_{1}} \mathcal{H} \eta_{x x}-\frac{\epsilon}{2} \eta_{x x x}=0 .
$$

This is the Benjamin equation. When capillarity can be neglected, the Benjamin-Ono equation

$$
\eta_{t}+\eta_{x}+\epsilon \frac{3}{2} \eta \eta_{x}-\epsilon \frac{1}{2} \frac{\rho_{2}}{\rho_{1}} \mathcal{H} \eta_{x x}=0
$$

appears (cf. [4,22]). We may again use the relation $\eta_{t}+\eta_{x}=O(\epsilon)$ in (3.6) to find the regularized Benjamin-Ono equation

$$
\eta_{t}+\eta_{x}+\epsilon \frac{3}{2} \eta \eta_{x}+\epsilon \frac{1}{2} \frac{\rho_{2}}{\rho_{1}} \mathcal{H} \eta_{t x}=0
$$

This equation has been under study in a number of articles (cf. $[2,16,17])$.

\section{Numerical simulation}

In order to conclude our discussion, we present a numerical simulation of the system (2.7) with $\epsilon=\sigma=\mu$, and a comparison with the Benjamin Eq. (3.5). Since both equations are formally valid in the same asymptotic regime, it is natural to ask whether solutions of these two equations stay close on some interval of time if the initial data are related by (3.4). Theorems in this direction have recently been proved in Ref. [1] for the regularized long-wave equation and a corresponding Boussinesq system modeling surface waves.

Let $\eta_{\mathrm{B}}(x, t)$ be the solution of the Benjamin Eq. (3.5), and let $\eta(x, t), w(x, t)$ be a solution of (2.7). Note that in this model, $\eta$ represents the deflection of the interface, while $w$ denotes the velocity of the fluid at the bottom. We are interested in the initial-value problem. That is, given initial data $w(x, 0)$ and $\eta(x, 0)$ at time 0 , the evolution according to (2.7) will be approximated numerically. For the purpose of comparison, we take as initial data

$$
\eta(x, 0)=\eta_{\mathrm{B}}(x, 0)
$$

and

$$
w(x, 0)=\eta_{\mathrm{B}}(x, 0)-\epsilon \frac{1}{4} \eta_{\mathrm{B}}^{2}(x, 0)-\epsilon \frac{1}{2} \frac{\rho_{2}}{\rho_{1}} \mathcal{H}\left(\eta_{\mathrm{B}}\right)_{x}(x, 0)-\epsilon \frac{1}{2}\left(\eta_{\mathrm{B}}\right)_{x x}(x, 0),
$$

as specified in (3.4). The discrepancy between $\eta$ and $\eta_{\mathrm{B}}$ will be called the error, and is defined by

$$
E(t)=\frac{\left\|\eta(\cdot, t)-\eta_{\mathrm{B}}(\cdot, t)\right\|_{N, 2}}{\|\eta(\cdot, t)\|_{N, 2}},
$$

where $\|\cdot\|_{N, 2}$ denotes the discrete $L^{2}$-norm, computed using a trapezoidal rule. If the theorems in Ref. [1] carry over to the situation at hand in the present paper, then we expect the estimate

$$
E(t) \leq C t,
$$

to be valid for $t \in[0, T / \epsilon]$ for some constants $C$ and $T$. In the sequel, this will be confirmed by numerical experiments in the case when $\epsilon=0.2$.

First, let us explain the method of numerical approximation to be used. Eq. (2.7) can be conveniently discretized in space using a Fourier-collocation method. For the time-stepping, a number of choices are available. We have used an Adams-Bashforth-Crank-Nicolson scheme which is second-order convergent. The advantage of this scheme is that the linear dispersive terms which contain high derivatives are handled implicitly, leading to an improved stability criterion. To save computational time, the nonlinear terms are computed explicitly. This procedure results in a conditionally stable, but fairly fast algorithm. Since no exact solutions of (2.7) are available, the convergence of the scheme cannot be verified directly. However, the convergence can be tested tested in other ways. Note that in the case when $\epsilon=\sigma=\mu$, the integral

$$
H(t)=\frac{1}{2} \int\left(w^{2}+\eta^{2}+\epsilon w^{2} \eta-\epsilon \frac{\rho_{2}}{\rho_{1}} \eta \mathcal{H} \eta_{x}+\epsilon \eta_{x}^{2}\right) \mathrm{d} x
$$


Table 1

Conservation of the functionals $H$ and $I$ : error between $H(0)$ and $H(1)$, and $I(0)$ and $I(1)$

\begin{tabular}{|c|c|c|c|c|c|}
\hline$N$ & $h$ & $H$-error & Ratio & $I$-error & Ratio \\
\hline $2^{8}$ & 0.01 & $3.06 \mathrm{e}-04$ & & $5.72 \mathrm{e}-05$ & \\
\hline $2^{8}$ & 0.005 & $7.12 \mathrm{e}-05$ & 4.30 & $1.36 \mathrm{e}-05$ & 4.22 \\
\hline $2^{8}$ & 0.0025 & $1.71 \mathrm{e}-05$ & 4.15 & $3.33 e-06$ & 4.06 \\
\hline $2^{8}$ & 0.0012 & $4.19 \mathrm{e}-06$ & 4.08 & $8.64 \mathrm{e}-07$ & 3.86 \\
\hline $2^{8}$ & 0.0006 & $1.04 \mathrm{e}-06$ & 4.05 & $2.58 \mathrm{e}-07$ & 3.36 \\
\hline $2^{8}$ & 0.0003 & $2.55 \mathrm{e}-07$ & 4.06 & $1.07 \mathrm{e}-07$ & 2.40 \\
\hline
\end{tabular}

Observe that the second order convergence of the temporal discretization is approximately achieved.

Table 2

Conservation of the functionals $H$ and $I$ : error between $H(0)$ and $H(1)$, and $I(0)$ and $I(1)$

\begin{tabular}{|c|c|c|c|c|c|}
\hline$N$ & $h$ & $H$-error & Ratio & $I$-error & Ratio \\
\hline $2^{5}$ & $2.0 \mathrm{e}-05$ & $3.21 \mathrm{e}-02$ & & $2.79 \mathrm{e}-01$ & \\
\hline $2^{6}$ & $2.0 \mathrm{e}-05$ & $1.68 \mathrm{e}-03$ & 19.1 & $9.49 \mathrm{e}-03$ & 29.4 \\
\hline $2^{7}$ & $2.0 \mathrm{e}-05$ & $3.07 \mathrm{e}-05$ & 54.9 & $7.52 \mathrm{e}-05$ & 126.2 \\
\hline $2^{8}$ & $2.0 \mathrm{e}-05$ & $1.85 \mathrm{e}-09$ & 1655.1 & $1.74 \mathrm{e}-07$ & 433.0 \\
\hline $2^{9}$ & $2.0 \mathrm{e}-05$ & $2.51 \mathrm{e}-10$ & 7.3 & $2.04 \mathrm{e}-10$ & 851.8 \\
\hline
\end{tabular}

Observe that the spectral convergence of the spatial discretization is approximately achieved.

is invariant in time if $w$ and $\eta$ are solutions of (2.7). In fact, (2.7) can be formally written as the Hamiltonian system

$$
\partial_{t} w=\left(\partial_{x}\right) \frac{\partial H}{\partial \eta}, \quad \partial_{t} \eta=\left(-\partial_{x}\right) \frac{\partial H}{\partial w} .
$$

A second invariant integral is given by the so-called impulse

$$
I(t)=\int u v \mathrm{~d} x
$$

The numerical implementation can therefore be validated by checking the conservation of these two functionals. A representative result is presented in Tables 1 and 2, where the values of $H(t)$ and $I(t)$ at time $t=1$ are compared with the values at time $t=0$. It is evident from these figures, that the expected convergence rate is achieved for both the time and the space discretization.

A second test on the numerical algorithm is done as follows. For a short time, a computation with an extremely fine time step is made. The computation can then be repeated with much coarser time steps, and by comparing these results with the first, an approximate convergence rate can be gleaned. The results of such an experiment are shown in Table 3.

After having validated the convergence of the numerical approximation, we are in a position to carry out a few numerical experiments. First, a Gaussian profile is used for the initial displacement of the interface, while the velocity was assumed to be initially zero. In Fig. 2, the evolution of the interface is shown, clearly exhibiting the bi-directional character of the equation (2.7).

Table 3

This is a computation with $N=128$

\begin{tabular}{lllll}
\hline$h$ & $L^{2}$-error in $w$ & Ratio & $L^{2}$-error in $\eta$ & Ratio \\
\hline 0.01 & $1.01 \mathrm{e}-04$ & & $6.42 \mathrm{e}-05$ & \\
0.005 & $2.50 \mathrm{e}-05$ & 4.03 & $1.59 \mathrm{e}-05$ & \\
0.0025 & $6.23 \mathrm{e}-06$ & 4.01 & $3.95 \mathrm{e}-06$ & 4.04 \\
0.0012 & $1.56 \mathrm{e}-06$ & 4.01 & $9.86 \mathrm{e}-07$ & 4.01 \\
0.0006 & $3.88 \mathrm{e}-07$ & 4.01 & $2.46 \mathrm{e}-07$ & 4.01 \\
0.0003 & $9.57 \mathrm{e}-08$ & 4.05 & $6.06 \mathrm{e}-08$ & 4.05 \\
\hline
\end{tabular}

Note that the second order convergence of the temporal discretization is also approximately achieved here. 

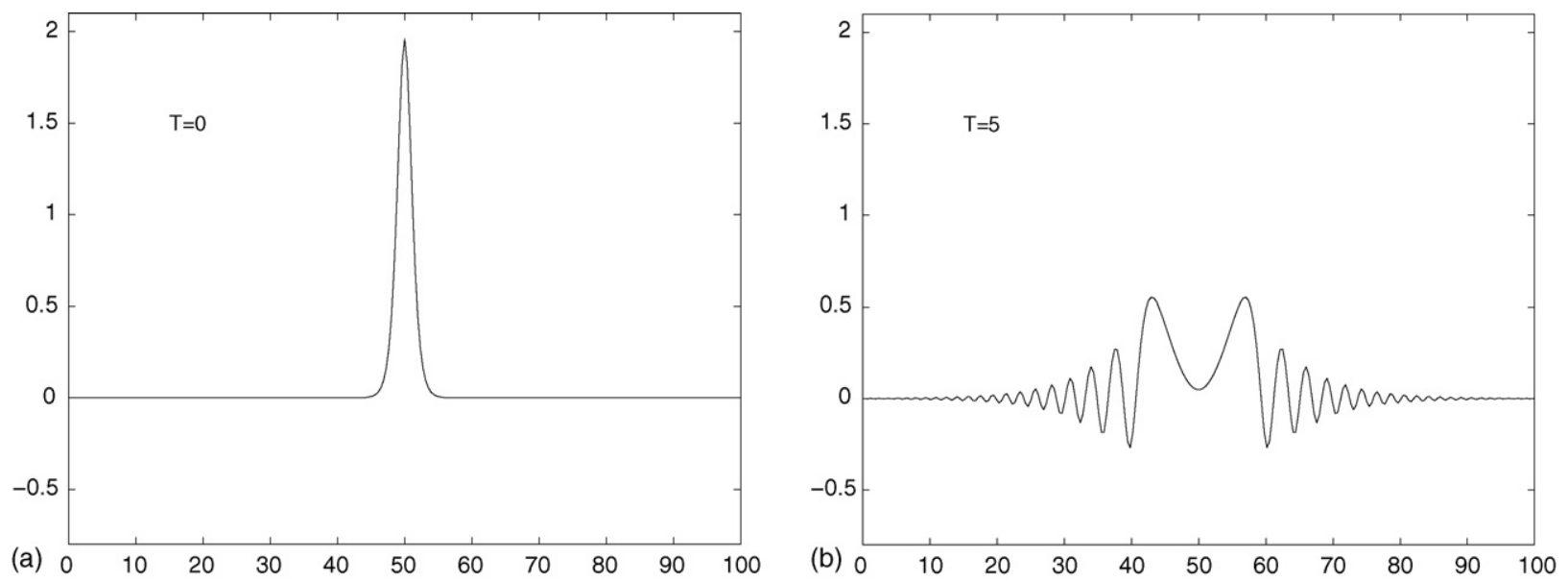

Fig. 2. Time evolution of the interface if initial data are given by a Gaussian, and the initial velocity is zero. The evolution clearly shows the bi-directional character of the equation.
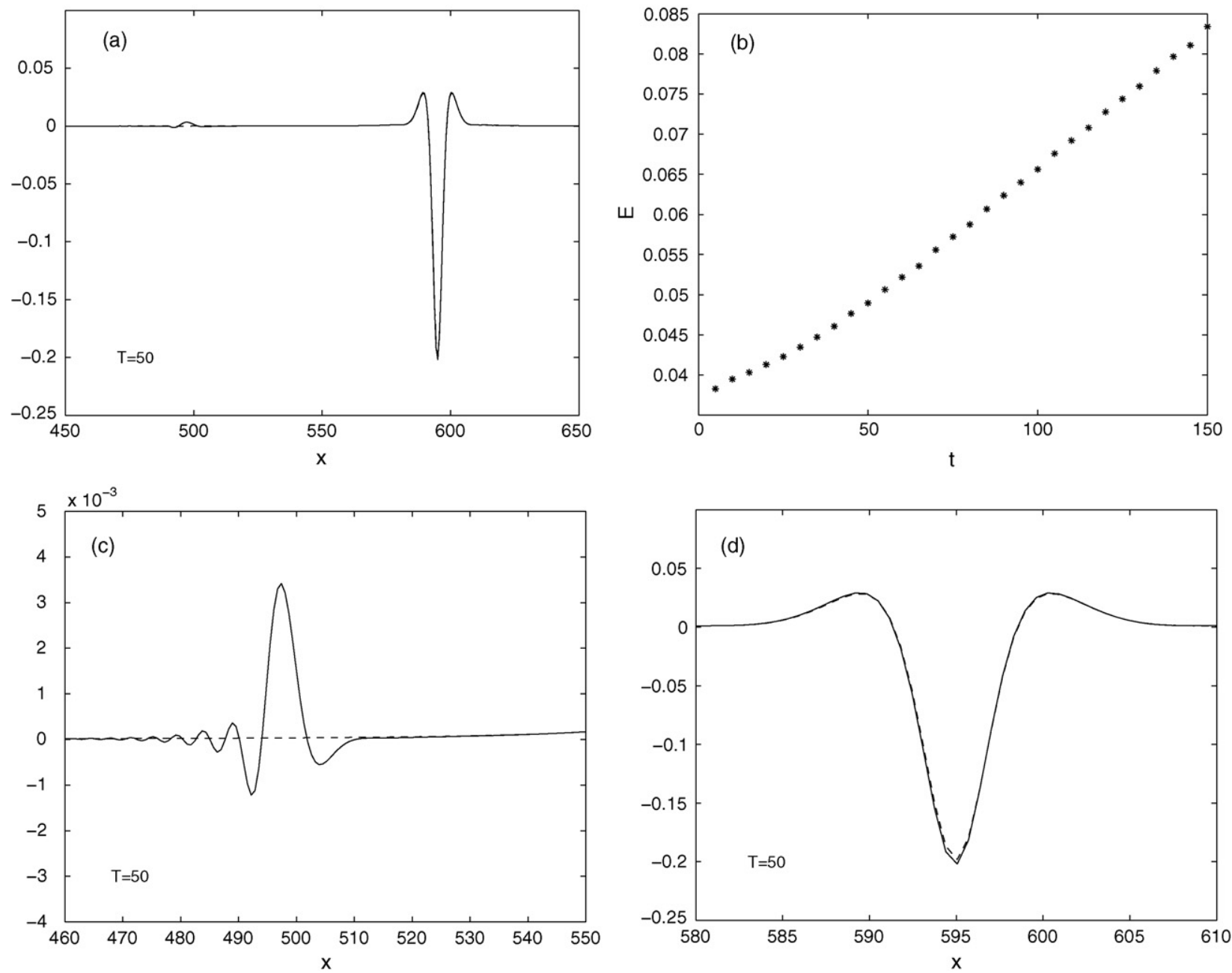

Fig. 3. (a) Time evolution of the interface if initial data are given by the solitary wave for the Benjamin equation. The solid line shows the interface profile according to Eq. (2.7), while the dashed line shows the evolution according to the Benjamin equation. (b) $E(t)$. (c) Close-up of the dispersive tail. (d) Close-up of the solitary wave. 

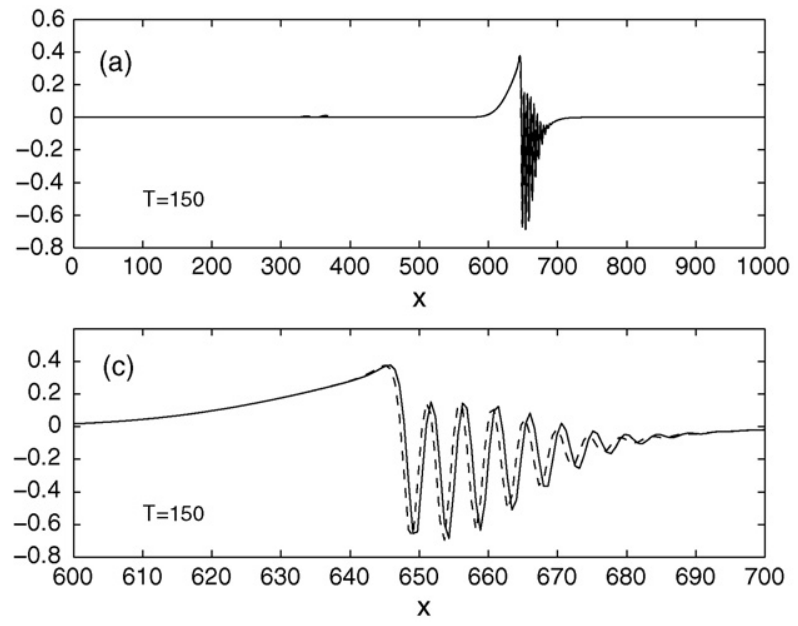

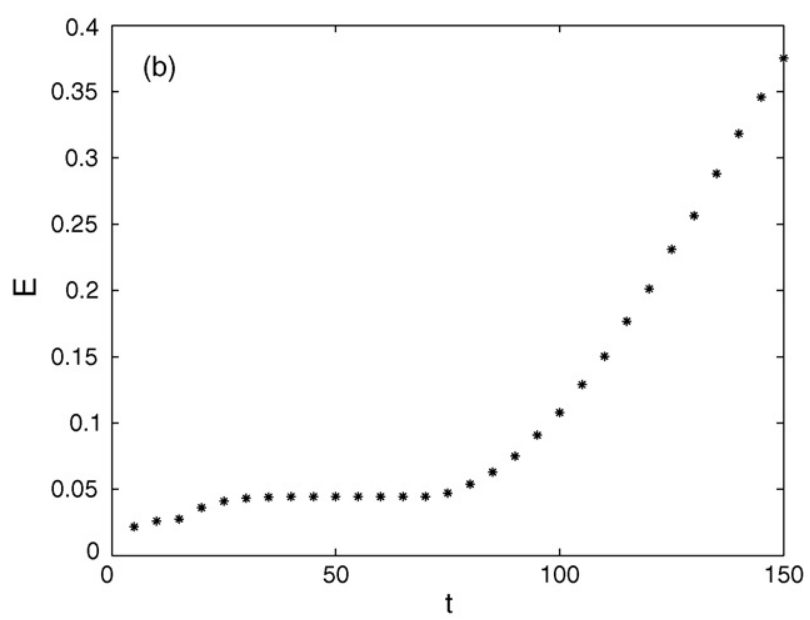

Fig. 4. (a) Time evolution of a dispersive wave. (b) $E(t)$. (c) Close-up: the solid line shows the interface profile according to Eq. (2.7), while the dashed line shows the evolution according to the Benjamin equation.

Next, the comparison of the system (2.7) with the Benjamin Eq. (3.5) is pursued. A numerical approximation to the Benjamin equation similar to the scheme just elucidated was explained in Ref. [17], where approximate solitary-wave solutions of the Benjamin equation were found. Solitary-wave solutions of (3.5) are of the form

$$
\eta_{\mathrm{B}}(x, t)=\phi(x-c t),
$$

where $c$ is the propagation speed. As it appears, solitary waves are steady wave profiles that translate without changing shape over time. Here, the evolution of these solitary waves in the Benjamin equation is compared with the evolution of corresponding solutions for (2.7). The initial data for (2.7) are constructed as follows. Suppose $\phi(x)$ is a solitary-wave profile for the Benjamin equation. Then the initial data for (2.7) are defined by

$$
\begin{aligned}
& \eta(x, 0)=\phi(x), \\
& w(x, 0)=\phi(x)-\epsilon \frac{1}{4} \phi(x)^{2}-\epsilon \frac{1}{2} \frac{\rho_{2}}{\rho_{1}} \mathcal{H} \phi_{x}(x)-\frac{\epsilon}{2} \phi_{x x}(x),
\end{aligned}
$$

where the use of the relation (3.4) is warranted, because the solitary-wave solutions of the Benjamin equation are propagating to the right. Of course, the resulting solutions of (2.7) are not solitary waves. However, as shown in Fig. 3, the solutions of (2.7) stay relatively close to the solitary wave of the Benjamin equation. In fact, it appears in Fig. 3(d) that the phase error between the two waves is negligible, and that except for a dispersive tail, a very good approximation of a solitary wave for (2.7) emerges. Moreover, it appears that this solitary wave propagates almost at the same speed as the solitary wave of the Benjamin equation. Thus, it is not surprising that the error $E(t)$ between $\eta$ and $\eta_{\mathrm{B}}$ increases rather slowly. In fact, it can be seen in Fig. 3(b) that $E(t)$ increases linearly as expected. It is also apparent that the rate of decrease, while still linear, increases as $t$ becomes larger than $O(1 / \epsilon)$.

Finally, we investigate the error in a general dispersive solution, where a modulated Gaussian profile was used as initial data for the Benjamin equation, and the initial data for (2.7) are constructed in the same way as before. It can be seen in Fig. 4(b) that the error first grows slowly and linearly, and then seems to level off. However, for large enough times, the growth rate increases again but is still linear. This seems to confirm in two special cases that the dependence of the error on the time $t$ is linear up to a time of $O(1 / \epsilon)$, and even beyond. As the equations in focus here are asymptotic models valid for $\epsilon \rightarrow 0$, it would be interesting to study the behaviour for smaller values of $\epsilon$. This question will be taken up in a subsequent article.

\section{Acknowledgement}

This work was supported in part by the BeMatA program of the Research Council of Norway. 


\section{References}

[1] A.A. Alazman, J.P. Albert, J.L. Bona, M. Chen, J. Wu, Comparisons between the BBM equation and a Boussinesq system, Adv. Differential Equations 11 (2006) 121-166.

[2] J.P. Albert, J.L. Bona, Comparisons between model equations for long waves, J. Nonlinear Sci. 1 (1991) 345-374.

[3] J.P. Albert, J.L. Bona, J.M. Restrepo, Solitary-wave solutions of the Benjamin equation, SIAM J. Appl. Math. 59 (1999) $2139-2161$.

[4] T.B. Benjamin, Internal waves of permanent form in fluids of great depth, J. Fluid Mech. 29 (1967) 559-592.

[5] T.B. Benjamin, A new kind of solitary wave, J. Fluid Mech. 245 (1992) 401-411.

[6] T.B. Benjamin, J.L. Bona, J.J. Mahony, Model equations for long waves in nonlinear dispersive systems, Philos. Trans. R. Soc. London Ser. A 272 (1972) 47-78.

[7] J.L. Bona, T. Colin, D. Lannes, Long wave approximations for water waves, Arch. Ration. Mech. Anal. 178 (2005) $373-410$.

[8] W. Choi, R. Camassa, Weakly nonlinear internal waves in a two-fluid system, J. Fluid Mech. 313 (1996) 83-103.

[9] W. Choi, R. Camassa, Fully nonlinear internal waves in a two-fluid system, J. Fluid Mech. 396 (1999) 1-36.

[10] P. Christodoulides, F. Dias, Stability of capillary-gravity interfacial waves between two bounded fluids, Phys. Fluids 7 (1995) $3013-3027$.

[11] W. Craig, An existence theory for water waves and the Boussinesq and Korteweg-de Vries scaling limits, Commun. Partial Differential Equations 10 (1985) 787-1003.

[12] W. Craig, P. Guyenne, H. Kalisch, A new model for large amplitude long internal waves, Comput. Rend. Mech. 332 (2004) 525-530.

[13] W. Craig, P. Guyenne, H. Kalisch, Hamiltonian long-wave expansions for free surfaces and interfaces, Commun. Pure Appl. Math. 58 (2005) $1587-1641$.

[14] F. Dias, G. Iooss, Capillary-gravity interfacial waves in infinite depth, Eur. J. Mech. B Fluids 15 (1996) 367-393.

[15] D.G. Ebin, Ill-posedness of the Rayleigh-Taylor and Helmholtz problems for incompressible fluids, Commun. Partial Differential Equations 13 (1988) 1265-1295.

[16] H. Kalisch, Error analysis of spectral projections of the regularized Benjamin-Ono equation, BIT 45 (2005) 69-89.

[17] H. Kalisch, J.L. Bona, Models for internal waves in deep water, Disc. Cont. Dyn. Syst. 6 (2000) 1-19.

[18] C.G. Koop, G. Butler, An investigation of internal solitary waves in a two-fluid system, J. Fluid Mech. 112 (1981) $225-251$.

[19] O. Laget, F. Dias, Numerical computation of capillary-gravity interfacial solitary waves, J. Fluid Mech. 349 (1997) $221-251$.

[20] H. Lamb, Hydrodynamics, Dover, New York, 1945.

[21] Y. Matsuno, A unified theory of nonlinear wave propagation in two-layer fluid systems, J. Phys. Soc. Jpn. 62 (1993) $1902-1916$.

[22] H. Ono, Algebraic solitary waves in stratified fluids, J. Phys. Soc. Jpn. 39 (1975) 1082-1091.

[23] L.A. Ostrovsky, J. Grue, Evolution equations for strongly nonlinear internal waves, Phys. Fluids 15 (2003) 2934-2948.

[24] G. Schneider, C.E. Wayne, The long-wave limit for the water wave problem. I. The case of zero surface tension, Commun. Pure Appl. Math. 53 (2000) 1475-1535.

[25] G. Schneider, C.E. Wayne, The rigorous approximation of long-wavelength capillary-gravity waves, Arch. Ration. Mech. Anal. 162 (2002) 247-285.

[26] H. Sha, J.-M. Vanden-Broeck, Internal solitary waves with stratification in density, J. Aust. Math. Soc. Ser. B 38 (1997) $563-580$.

[27] E.O. Tuck, L.H. Wiryanto, On steady periodic interfacial waves, J. Eng. Math. 35 (1999) 71-84.

[28] G.B. Whitham, Linear and Nonlinear Waves, Wiley, New York, 1974. 